\title{
Sobre as Bases de Referência Celeste
}

\author{
On the Celestial Reference Frames
}

\author{
P.C.R. Poppe* e V.A.F. Martin ${ }^{\dagger}$ \\ Departamento de Física - UEFS \\ Campus Universitário, Km 03 BR 116 \\ Feira de Santana - BA - 44031-460
}

\begin{abstract}
Ao longo deste artigo, apresentamos, como continuidade à discussão apresentada no trabalho anterior [1], uma primeira discussão sobre algumas das principais bases de referência utilizadas em Astronomia. Da mesma forma, esperamos que o presente texto possa ser utilizado ao longo dos cursos de Introdução à Astronomia das atuais graduações de Física e áreas afins.
\end{abstract}

Palavras-chaves: Introdução à Astronomia, Bases de Referência, Graduação em Física.

In connection to the paper [1], we briefly describe in the present work some discussions about the Celestial Reference Frame in Astronomy. First we have chosen, for practical reasons, the Basic FK4, FK5, IERS and Hipparcos. We hope that the material presented may be usefully employed in the introductory courses of Astronomy, which are usually offered in the undergraduate curriculum of physics.

Key-words: Introduction to Astronomy, References Frames, Physics Undergraduation.

\section{INTRODUÇÃO}

Já vimos [1] que o movimento é um conceito relativo e que deve ser sempre referido a um referencial específico, escolhido pelo observador. Como diferentes observadores podem usar diferentes referenciais, torna-se imperativo e de grande importância saber relacionar todas as observações envolvidas. Na prática, um experimentador normalmente escolhe um referencial em relação ao qual as tomadas de dados, bem como as posteriores análises, possam ser realizadas de maneira mais fácil possível. Por exemplo, a maioria das observações realizadas na Terra são referidas à referenciais ligados à própria Terra e que se movem com ela; em Física Atômica, o movimento dos elétrons é determinado relativamente ao núcleo do átomo; já em Astronomia, busca-se materializar um sistema de referência o mais inercial possível, isto é, sem acelerações ou rotações residuais, de forma a referenciar um dado objeto celeste em estudo. Atualmente, podemos dizer que o descobrimento dos quasares e das radiofontes extragalácticas compactas com contrapartida óptica, associados à precisão conseguida por novas técnicas de rádio-interferometria, possibilitaram a construção de um conjunto de pontos fiduciais (eixos inerciais), cujo movimento próprio pode ser considerado nulo, ou pelo menos desprezível. Este aspecto ainda constitui um dos principais objetivos da Astrometria.

No que segue (seção 2), traçaremos um pequeno panorama histórico quanto a evolução de alguns dos principais catálogos utilizados ao longo do tempo pela Astronomia, reservando para a seção 3 os catálogos $F K 4$ e $F K 5$. $\mathrm{Na}$ verdade, as bases de referência são freqüentemente representadas por catálogos de estrelas, mas podem igualmente ser representadas por uma efeméride cobrindo um período suficientemente longo, que pode ser representada

\footnotetext{
*Endereço Eletrônico: poppe@uefs.br

$\dagger$ Endereço Eletrônico: vmartin@uefs.br
}

pelos planetas principais e pela Lua. Podemos apontar, como exemplo, as integrações numéricas do Jet Propulsion Laboratory DE200/LE200 [2].

Em tempo, vale a pena ressaltar que o significado de catálogo discutido neste trabalho ultrapassa a simples definição de uma "lista de objetos com dados astrométricos, fotométricos, espectroscópicos, etc.".

\section{UM HISTÓRICO NECESSÁRIO}

\section{A. De Hiparcos à Bessel}

Dentre os vários trabalhos realizados por Hiparcos (século II a.C.), salientamos aquele referente à elaboração de um catálogo estelar, constituído pelas posições em coordenadas eclípticas de aproximadamente 1000 estrelas. Neste, as posições eram conhecidas com uma precisão de $\pm 30^{\prime}$ e divididas em classes de magnitude, de acordo com a luminosidade observada. Certamente, apesar da qualificação, este catálogo foi suficientemente preciso para descrever a compreensão do universo em debate naquela época [3].

Sucessivas contribuições ${ }^{1}$, ainda que bem espaçadas no

1 Uma das particularidades do trabalho de Hiparcos é certamente a descoberta da precessão dos equinócios, comparando a longitude de Vir (Spica) com àquela determinada anteriormente ( 144 anos) por Timocharis e Aristillos. Mais tarde, já na era cristã, Claudio Ptolomeu ( 150 d.C, em sua obra essencial, o "Almagesto") retoma as observações efetuadas por Hiparcos e leva em consideração a precessão dos equinócios. Bem mais tarde, as observações realizadas por Tycho Brahe (1600) e por Johannes Hevelius (1680) forneceram, pela primeira vez, em coordenadas equatoriais, as posições de aproximadamente 1500 estrelas (com uma precisão de $\left.\pm 2^{\prime}\right)$, retocando definitivamente a esfera das fixas que ficara prati- 
tempo, foram imprescindíveis para que um importante passo na elaboração dos catálogos estelares fosse dada no século XVIII por Halley (1718) e Cassini (1738), isto é, a existência dos movimentos próprios, que seriam confirmados posteriormente, para um conjunto aproximado de 60 estrelas, por Tobias Mayer (em Göttingen) e por Bradley e Maskelyne (em Greenwich).

Com relação a Bradley, vale lembrar que, apesar das quase 60000 observações realizadas de aproximadamente 3200 estrelas (com uma precisão de $\pm 2^{\prime}$ ) entre 1750 e 1765, coube a Bessel a função de reduzir tais observações e reagrupá-las em 1818 no "Fundamenta Astronomiae". Estas estrelas, entretanto, não puderam fornecer de maneira direta (ou seja, materializar) um sistema de referência, uma vez que os movimentos próprios destes objetos não haviam sido determinados de maneira satisfatória. $\mathrm{Na}$ verdade, podemos dizer que este catálogo proporcionou um sistema de coordenadas para a época média $t=1755$.

Estes fatos, ainda que apresentados muito brevemente, foram capitais para consolidar a posteriori construção dos inúmeros catálogos estelares que conhecemos atualmente. O leitor poderá encontrar na recente publicação de Walter e Sovers [4], bem como na vasta literatura contida, uma excelente discussão sobre Astrometria e Catálogos Fundamentais

\section{B. De Bessel à HIPPARCOS}

Motivado pelas observações zodiacais de Bradley e Maskelyne (36 estrelas com o objetivo de estabelecer uma base de referência), Bessel completa tais observações entre 1825 a 1830 no Observatório de Königsberg e publica o assim chamado "Tabulae Regiomantanae", que fornecia, além das posições aparentes das estrelas zodiacais de 1750 a 1850 , um novo valor para a precessão. Aqui, contrariamente ao discutido acima, estes resultados serviram de sistema de referência por quase cinqüenta anos.

O século avança, até que Newcomb, em 1872, determina, a partir de diferentes observações das mesmas estrelas discutidas por Bradley, agora presentes no "Right Ascensions of the Equatorial Stars", um valor para o equinócio, conhecido na literatura como $N 1$. Este valor foi adotado em 1882 para o "Catalogue of 1098 Standard Clock and Zodiacal Stars" e em 1899 para o "Fundamental Catalogue". Podemos encontrar no catálogo de Newcomb, comparações de catálogos estabelecidos entre 1830 e 1880 que colocavam em evidência um movimento suplementar do equinócio, isto é, uma "precessão" proveniente de efeitos sistemáticos durante as observações (equações pessoais durante o registro do trânsito de uma estrela ou do limbo de um planeta, entre uma observação do dia ou da noite, ou segundo a metodologia de registro do instante do trânsito). Na verdade, Newcomb suspeitou que o observador registrava o trânsito de uma estrela fraca muito tarde e aquela, de uma estrela brilhante, muito cedo, sendo confirmado mais tarde por Küstner em 1902 [5]. Com Kahrstedt em 1931 [6], aprendemos a "evitar" este erros sistemáticos, mas que, segundo

camente inalterada por quase 1500 anos. Na mesma época, Isaac Newton (1680) explica a precessão dos equinócios baseada na ação luni-solar sobre o bojo equatorial da Terra.
Fricke, em 1985 [7], "os observadores não o fazem devidamente". Outros catálogos reagrupando um número maior de estrelas fracas surgem na segunda metade do século XIX, como o N30 de Morgan e o CG de Boss. Claramente, uma maior acessibilidade destes à objetos fracos está intimamente ligada ao detrimento de uma representação mais precisa de um sistema de referência.

Em 1879, Auwers começa com o " $F K$ of the Northern Sky" (Fundamentalkatalog für die Zonen Beobachtungen am Nördlichen Himmel), a construção de uma série de catálogos alemãs. Este catálogo compreendia somente 539 estrelas de declinação superior a $-10^{\circ}$ e serviu de sistema de referência para o $A G K 1$ (Astronomische Geselltschaft Katalog). Este, por sua vez, cobria somente o Hemisfério Norte e foi complementado em 1907 pelo NFK de Peters (Neuer Fundamentalkatalog des Berliner Astronomischen Jahrbuchs nach den Grundlagen von A. Auwers) que possuía 925 estrelas. O equinócio $N 1$ e o valor da precessão estabelecida por Newcomb foram devidamente aplicados. Este catálogo representou uma extensão do $F K$, onde a precisão sobre as posições e movimentos próprios das estrelas foi melhorada, além da inclusão de uma porção maior do Hemisfério Sul.

Em 1937, August Kopff publica o FK3 (Dritter Fundamentalkatalog des Berliner Astronomischen Jahrbuchs), formado por 1535 estrelas de magnitudes inferior a 7,5. Este catálogo serviu de referência para o $A G K 2$, com o equinócio determinado por Kahrstedt [6], correção do equinócio $N 1$.

Em 1938, a Assembléia Geral da IAU resolve que, a partir de 1940, as posições das estrelas utilizadas para a medida do tempo ou em conexão com os sinais rádios, seriam ligadas ao sistema $F K 3$. Vinte anos após a publicação deste sistema, 72 novos catálogos estelares foram construídos e a IAU recomenda então a construção do $F K 4$, publicado em 1963 por Fricke e Kopff [8], consistindo de uma revisão do $F K 3 \mathrm{com}$ as correções individuais (determinadas a partir da comparação do $F K 3$ com outros catálogos) nos movimentos próprios e nas posições. Este novo catálogo incluía observações de objetos do Sistema Solar (Sol, Lua e planetas), uma correção ao equinócio [9] e uma correção para ao equador [8]. Contudo, duas grandes razões levaram a substituição do $F K 4$, dez anos após a sua construção:

a) os erros nos movimentos próprios das estrelas do $F K 4$ diminuíam sensivelmente a precisão sobre as posições na época J2000 (em particular para as estrelas do Hemisfério Sul que foram observadas posteriormente, ou mais tardiamente);

b) as técnicas e as precisões nas observações colocaram em evidência erros zonais e sistemáticos do $F K 4$.

Desse modo, a IAU recomendou em 1973 a melhoria do FK 4 e a sua extensão à estrelas mais fracas. Seu "defeito" mais importante estava no movimento errôneo de seu ponto vernal, o que levou a um erro sistemático nos movimentos próprios (utilizados em dinâmica estelar ou para a rotação da galáxia). O novo sistema, $F K 5$, [10] deveria, portanto, corrigir o equinócio e o equador do $F K 4$ [11], levando em conta a constante de precessão adotada pela IAU em 1976 [12]. Este sistema é ligeiramente diferente das soluções adotadas no passado: seguindo uma análise dos movimentos próprios, ele contém, além da correção da precessão, uma determinação da rotação do campo de estrelas próximas induzidas pela rotação da galáxia. 
Do exposto anteriormente, podemos concluir que os movimentos próprios das estrelas fundamentais são bem menos determinadas que suas posições para uma dada época, o que acaba limitando a vida útil de um catálogo fundamental (cerca de 25 anos) e explica o porque a cada construção de catálogo, é preciso pensar desde já em um sucessor. Por outro lado, várias outras limitações ligadas as observações a partir do solo devem ser lembradas durante a construção de um novo catálogo (atmosfera, movimento do pólo, cobertura parcial do céu, etc.).

De um modo geral, os catálogos estelares representam mal a vizinhança dos pólos celestes e de maneira não homogênea os dois hemisférios. Além dos mais, eles comportam erros zonais oriundos de erros sistemáticos provenientes de diferentes instrumentos utilizados para a compilação. Exemplos podem ser dados: o catálogo PPM [13] e o próprio $F K 5$ [10], uma importante base de referência dinâmica [14].

Diante desses aspectos, parece-nos claro que uma missão observacional astrométrica espacial representaria a solução. Na verdade, esta proposição não é uma idéia muito recente: Pierre Lacroûte, astrônomo francês, apresentava, nos anos 60 [15], tal proposta para apreciação na Agência Espacial Européia (ESA). Todavia, recente foi a conclusão desta idéia $^{2}$, três décadas após, colocando finalmente o primeiro satélite astrométrico em órbita na Terra.

Iniciamos a Seção 2 citando um dos maiores astrônomos gregos que viveu em Rodes no século II a.C. e que deixou uma razoável base de conhecimento para os seus sucessores. Elaborou o primeiro catálogo estelar, determinou o comprimento do ano trópico, o tamanho e a distância da Lua, bem como as irregularidades no movimento; descobriu a precessão dos equinócios e aperfeiçoou instrumentos astronômicos. Todos estes resultados deste fabuloso trabalho foram preservados no Almagest de Ptolomeu.

Nesse sentido, parece-nos muito justa a homenagem feita a este personagem grego, guardando-lhe o nome do satélite astrométrico discutido linhas acima: HIPPARCOS, acrônimo da expressão inglesa "High Precision Parallax Collecting Satellite" (Satélite de Coleta de Paralaxe de Alta Precisão), missão esta que forneceu até o término das operações (em 15 de Outubro de 1993), posições, paralaxes e movimentos próprios de aproximadamente 120 mil estrelas. Finalmente, esta catálogo possui, além de sua fantástica precisão, duas outras qualidades: ele é homogêneo e os objetos são melhor repartidos ao longo da esfera celeste [16].

\section{AS BASES DE REFERENCIA CELESTE}

\section{A. O FK4 $(B 1950,0)$}

A base do FK4 (Fundamental Katalog 4, Fricke e Kopff 1963 [8]) é representada pelas coordenadas equatoriais

2 O satélite foi lançado em 08 de Agosto de 1989, descrevendo uma órbita elíptica ao redor da Terra com uma excentricidade de 0,72 e um período de revolução de $10^{h} 40^{m}$. médias $(\alpha, \delta)$ e pelos movimentos próprios $\left(\mu_{\text {alpha }}, \mu_{\text {delta }}\right)$ de 1535 estrelas (além de um catálogo suplementar de 1987 estrelas), com época de referência B1950, 0. Neste catálogo, bem como naqueles anteriores a 1984, todas as coordenadas estão afetadas pelos termos de aberração elíptica.

O plano equatorial médio de $B 1950,0$, com uma precisão estimada de $0^{\prime \prime}, 02$, representa o plano de referência para este sistema. Quanto ao equinócio, de acordo com Fricke 1982 [11], este representa o equinócio dinâmico médio rotacional de $B 1950,0$ com um erro de aproximadamente $0^{s}, 035\left(=0^{\prime \prime}, 525\right)$.

O sistema FK4 é compatível com as teorias ou as expressões de Newcomb para o Sol, os planetas, a precessão e o tempo sideral. Para a precessão geral em longitude, utilizada para sua construção, temos:

$$
p_{N}=5025^{\prime \prime}, 64 \text { por século trópico }(B 1900,0) \text {, }
$$

e para a obliqüidade (rotacional, segundo Standish (1982) $[17])$ :

$$
\varepsilon_{N}^{R}=23^{\circ} 27^{\prime} 8^{\prime \prime}, 26 \quad(B 1900,0) .
$$

\section{B. O FK5 $(J 2000,0)$}

Este sistema (Fundamental Katalog 5, Fricke et al. 1988 [10]), com época de referência $J 2000,0$, é representado pelas mesmas estrelas presentes no sistema $F K 4$, mas agora sem os termos de aberração elíptica. As precisões para este sistema, segundo Kovalevsky 1989 [18], são estimadas em $0^{\prime \prime}, 02$ em posição e $0^{\prime \prime}, 08$ por século em movimento próprio. O plano de referência representa o plano equatorial médio de $J 2000,0$, com uma precisão estimada ${ }^{3}$ de $0^{\prime \prime}, 02$. De acordo com Fricke [11], o equinócio representa o equinócio dinâmico médio rotacional de $J 2000,0$ com uma precisão da ordem de $0^{s}, 003\left(=0^{\prime \prime}, 045\right)$.

O catálogo $F K 5$ é compatível com as expressões de Lieske para a precessão [12] e as de Aoki para o tempo sideral [19], repousando ambas na teoria de Newcomb para o Sol, mas com valores diferentes para a massa. O valor da precessão geral em longitude vale:

$$
p_{L}=5029^{\prime \prime}, 0966 \text { por século juliano }(J 2000,0),
$$

com o correspondente valor 5026", 767 por século trópico em B1900, 0.

Podemos verificar que a diferença com a constante de Newcomb (de $1^{\prime \prime}, 127$ por século trópico), resulta de uma correção na precessão luni-solar de $1^{\prime \prime}, 10 \pm 0^{\prime \prime}, 15$ por século trópico, determinada por Fricke [20] e de uma correção na precessão planetária calculada por Lieske et al. 1977 [12].

O valor da obliqüidade em $J 2000,0$, para uma eclíptica rotacional, associada ao sistema $F K 5$, vale:

$$
\varepsilon_{0}^{R}=23^{\circ} 26^{\prime} 21^{\prime \prime}, 448 .
$$

Esta obliqüidade é deduzida do valor de Newcomb B1900, 0 por meio das expressões da precessão de Lieske et al. 1977 [12]. Ela resulta de recentes determinações da

3 De acordo com Arias et al. 1991 [21], o erro real é de 2 a 5 vezes maior que o erro sobre a constante de precessão. 
obliqüidade da eclíptica dinâmica média J2000,0, onde a eclíptica média $J 2000,0$ associada ao $F K 5$ difere da eclíptica dinâmica média rotacional em J2000, 0 (ver Figura 1). Um valor aproximado do ângulo entre os dois planos vale:

$$
\triangle \varepsilon=0^{\prime \prime}, 036
$$

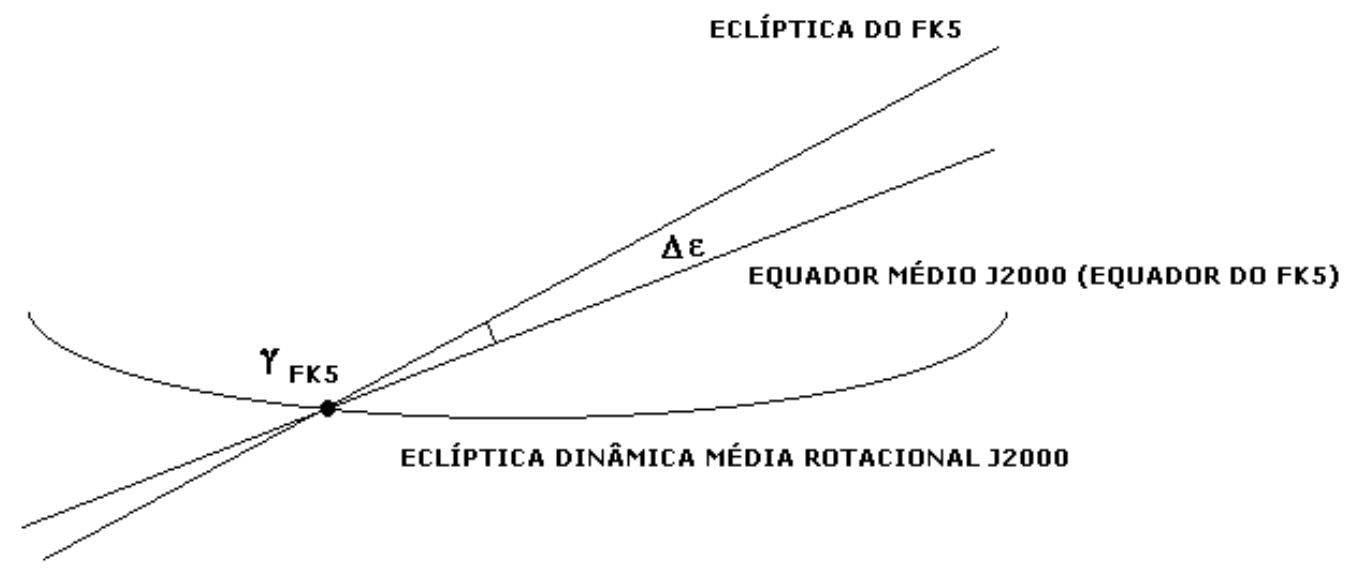

Fig. 1 - Eclíptica do FK5 e Eclíptica Dinâmica

O FK5 representou o sistema convencional adotado pela União Astronômica Internacional em 1976, sendo sua efetiva utilização nas efemérides astronômicas a partir de 1984.

\section{RELAÇÕES ENTRE BASES DE REFERÊNCIA}

No que segue, iremos apresentar, de maneira não exaustiva, alguns métodos construídos para relacionar, sobretudo, as bases do $F K 4$ e do $F K 5$. Relações destas com outras bases, tais como aquelas construídas pelo JPL ou pelo $B D L$, serão discutidas em uma outra contribuição.
Dentro do trabalho de Lieske et al. 1977 [12], encontramos a transformação (de precessão) que permite deduzir o plano de referência do $F K 5$ a partir do plano de referência do $F K 4$, entre $B 1950,0$ e J2000, 0 . Por outro lado, a transformação necessária para passar do equinócio do $F K 4$ para o equinócio do $F K 5$ é mais complicada e dá lugar à diferentes interpretações. Segundo Fricke 1982 [11], temos a seguinte expressão:

$$
E(T)=0^{s}, 035 \pm 0^{s}, 003+\left(0^{s}, 085 \pm 0^{s}, 010\right)(T-19,50)
$$

A. Passagem do $F K 4$ para o $F K 5$

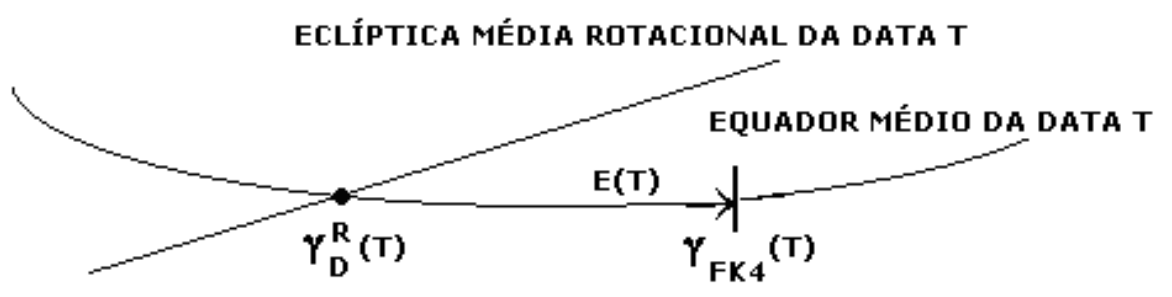

Fig. 2 - Passagem do Equinócio do $F K 4$ ao Equinócio do $F K 5$

que de acordo coma a Figura 2, representa o arco $\gamma_{D}^{R}(T) \hat{\gamma}_{F K 4}(T)$ entre o equinócio dinâmico médio rotacional da data $T$ (em séculos), $\gamma_{D}^{R}(T)$, e o equinócio médio da data $T$ ligado ao sistema $F K 4, \gamma_{F K 4}(T)$. Este arco é medido ao longo do equador médio da data $T$, que supomos idêntico ao equador correspondente ligado ao $F K 4$. Não devamos esquecer, entretanto, que os dois sistemas são ligados à diferentes constantes de precessão. Na prática, para passar das coordenadas dos astros presentes no $F K 4$ para as coordenadas no $F K 5$, podemos distinguir vários métodos, segundo estrelas dotadas de movimentos próprios, radiofontes, planetas ou satélites. 


\section{B. A Construção do $F K 5$ a partir do $F K 4$}

Encontramos, dentro dos trabalhos de Fricke 1982a [22] e Fricke et al. 1988 [10], a maneira que nos permite passar para o $F K 5$, as coordenadas e movimentos próprios publicados no $F K 4$ :

a) em primeiro lugar, acrescentamos nas ascensões retas das estrelas publicadas no $F K 4(1950,0)$, a correção de equinócio $E(19,50)=0^{s}, 035$, e nos movimentos próprios em ascensão reta, a correção $0^{s}, 085$ por século ( $=1^{\prime \prime}, 275$ por século);

b) em segundo lugar, a mudança de valor da precessão geral em longitude é levada em conta acrescentando aos movimentos próprios, em ascensão reta e em declinação, as correções ${ }^{4}$

$$
\begin{aligned}
& \triangle \mu_{\alpha}=-1^{\prime \prime}, 03-0^{\prime \prime}, 44 \sin \alpha \tan \delta \\
& \triangle \mu_{\delta}=0^{\prime \prime}, 44 \cos \alpha
\end{aligned}
$$

c) finalmente, após adição das correções sistemáticas (funções de $\delta$ e $\alpha$ ) e eliminação da aberração elíptica, o conjunto de coordenadas e de movimentos próprios corrigidos em $B 1950,0$ é levado ao equador e equinócio médios de J2000,0 com ajuda das expressões da precessão de Lieske et al. 1977 [12]; as coordenadas foram calculadas em J2000, 0 com a ajuda dos movimentos próprios e eventualmente da velocidade radial e da paralaxe.

\section{Caso: Estrelas Quaisquer}

Neste caso, o objetivo consiste em calcular, para estrelas que não fazem parte dos catálogos $F K 4$ e $F K 5$, a ascensão reta, a declinação e os respectivos movimentos próprios em J2000, 0 levados ao plano de referência e ao equinócio do FK5 (precessão de Lieske), em função das coordenadas $\alpha_{0}$ e $\delta_{0}$ e dos movimentos próprios $\mu_{\alpha, 0}$ e $\mu_{\delta, 0}$ destas estrelas em $B 1950,0$, com plano de referência e equinócio do $F K 4$ (precessão de Newcomb). Vamos considerar que os movimentos próprios estejam expressos em "/século trópico no sistema $F K 4$ e em " / século juliano no sistema $F K 5$.

Ao longo da década de 80, vários métodos foram publicados para resolver este problema. No que segue, vamos expor os métodos de Standish (1982) [23], de Aoki et al. [19] e de Murray [24], e que possuem em comum os seguintes pontos:

a) os vetores "posição" e "velocidade" no sistema $F K 4$

4 Em primeira aproximação, as correções aos movimentos próprios devidos a uma mudança da precessão são da forma $\triangle \mu_{\alpha}=-(\triangle m+$ $\triangle n \sin \alpha \tan \alpha) \triangle \mu_{\delta}=-\triangle n \cos \alpha$, onde $\mathrm{m}$ e $\mathrm{n}$ são as modificações, respectivamente, nas velocidades de precessão geral em ascensão reta e em declinação.
$(B 1950,0)$, podem ser calculados da seguinte forma:

$$
\begin{aligned}
\vec{r}_{0}= & \left(\begin{array}{c}
\cos \alpha_{0} \cos \delta_{0} \\
\sin \alpha_{0} \cos \delta_{0} \\
\sin \delta_{0}
\end{array}\right) \\
\vec{v}_{0}= & \left(\begin{array}{c}
-\left(\sin \alpha_{0} \cos \delta_{0}\right) \mu_{\alpha, 0}-\left(\cos \alpha_{0} \sin \delta_{0}\right) \mu_{\delta, 0} \\
\left(\cos \alpha_{0} \cos \delta_{0}\right) \mu_{\alpha, 0}-\left(\sin \alpha_{0} \sin \delta_{0}\right) \mu_{\delta, 0} \\
\left(\cos \delta_{0}\right) \mu_{\delta, 0}
\end{array}\right) \\
& +21,094502 \pi_{0} V_{0} \vec{r}_{0}
\end{aligned}
$$

onde $V_{0}$ representa a velocidade radial $\mathrm{em} \mathrm{km} / \mathrm{s}$ e $\pi_{0}$ a paralaxe em ", no sistema $F K 4$. Se alguma destas quantidades for desconhecida, ela será suposta nula. $\vec{r}_{0}$ não possui dimensão e $\vec{v}_{0}$ é expresso em "/século trópico. O coeficiente numérico 21,094502 representa o quociente do comprimento do século trópico em segundos pelo comprimento da unidade astronômica em $\mathrm{km}$.

b) os termos de ordem da aberração elíptica podem ser eliminados calculando-se os vetores:

$$
\begin{aligned}
& \vec{r}_{1}=\vec{r}_{0}-\Delta \vec{r}_{0} \\
& \vec{v}_{1}=\vec{v}_{0}-\Delta \vec{v}_{0}
\end{aligned}
$$

c) o vetor "posição" (sem dimensão) e o vetor "velocidade" (em "/século juliano) no sistema $F K 5(J 2000,0)$ são calculados por:

$$
\left(\begin{array}{l}
\vec{r} \\
\vec{v}
\end{array}\right)=\left(\begin{array}{c}
x \\
y \\
z \\
\dot{x} \\
\dot{y} \\
\dot{z}
\end{array}\right)=M\left(\begin{array}{l}
\vec{r}_{1} \\
\vec{v}_{1}
\end{array}\right)
$$

onde $\mathrm{M}$ é uma matriz $6 \times 6$ que pode ser decomposta em 4 matrizes $3 \times 3$, do tipo:

$$
M=\left(\begin{array}{ll}
M_{11} & M_{12} \\
M_{21} & M_{22}
\end{array}\right),
$$

d) com auxílio de (17), podemos escrever as seguintes expressões:

$$
\begin{aligned}
r & =\sqrt{x^{2}+y^{2}+z^{2}}, \\
\delta & =\sin ^{-1}(z / r), \\
x / r & =\cos \delta \cos \epsilon, \\
y / r & =\cos \delta \cos \alpha, \\
\mu_{\alpha} & =(x \dot{y}-y \dot{x}) /\left(x^{2}+y^{2}\right), \\
\mu_{\delta} & =\left(\dot{z} r^{2}-z \vec{r} \cdot \vec{v}\right) /\left(r^{2} \sqrt{x^{2}+y^{2}}\right), \\
\pi & =\pi_{0} / r, \\
V & =0,0474047047(\vec{r} \cdot \vec{v}) /\left(\pi_{0} r\right),
\end{aligned}
$$

onde $V$ representa a velocidade radial em $\mathrm{km} / \mathrm{s}$ e $\pi_{0}$ a paralaxe em ", no sistema $F K 5$. O coeficiente numérico 0,047404704 é o coeficiente do comprimento da unidade astronômica em $\mathrm{km}$ pelo comprimento do século juliano em segundos. 


\section{Método de Standish}

Este método é equivalente, em seu princípio, aos métodos descritos por Fricke [22] e por Fricke et al. 1988 [10] para a construção do $F K 5$, salvo que este não possui correções individuais. A aberração elíptica é eliminada com a ajuda das seguintes relações, onde $\times$ designa o produto vetorial:

$$
\begin{aligned}
\triangle \vec{r}_{0} & =\vec{r}_{0} \times\left(\vec{A} \times \vec{r}_{0}\right), \\
\triangle \vec{v}_{0} & =0,
\end{aligned}
$$

com

$$
A=10^{-6}\left(\begin{array}{c}
-1,62558 \\
-0,31920 \\
-0,13841
\end{array}\right) \text {, }
$$

\section{Método de Aoki}

Este representou o método preconizado pelo projeto MERIT (Melbourne 1983, [25]). Compreende as seguintes etapas:

a) as coordenadas equatoriais médias e os movimentos próprios $B 1950,0$ no sistema $F K 4$, são corrigidos da aberração elíptica e transformados em coordenadas equatoriais médias e movimentos próprios para 1984, 0 (data a qual o sistema $F K 5$ entrou efetivamente em vigor), levados ao equador e equinócio médios desta data, com ajuda da precessão de Newcomb e dos movimentos próprios;

b) as correções do equinócio e dos movimentos próprios são efetuadas sobre as coordenadas médias de 1984, 0;

c) as coordenadas e os movimentos próprios, assim obtidas, são transformados em coordenadas equatoriais médias e movimentos próprios J2000,0 no sistema FK5, com ajuda da precessão de Lieske et al. [12] entre 1984, 0 e $J 2000,0$ e dos movimentos próprios.

Recuperando as equações (9), a aberração elíptica pode ser eliminada com ajuda das seguintes relações:

$$
\begin{aligned}
& \Delta \vec{r}_{0}=\vec{A}-\left(\vec{r}_{0} \cdot \vec{A}\right) \vec{r}_{0}, \\
& \triangle \vec{v}_{0}=\dot{\vec{A}}-\left(\vec{r}_{0} \cdot \dot{\vec{A}}\right) \vec{r}_{0},
\end{aligned}
$$

onde

$$
\begin{aligned}
& A=10^{-6}\left(\begin{array}{c}
-1,62558 \\
-0,31920 \\
-0,13841
\end{array}\right), \\
& \dot{\vec{A}}=10^{-6}\left(\begin{array}{c}
+1,25 \\
-0,158 \\
-0,659
\end{array}\right) .
\end{aligned}
$$

As primeiras linhas das relações (13) e (15) são equivalentes.

\section{Método de Murray}

Este método é equivalente, dentro de seus princípios, ao método de Standish, mas a matriz $M$ difere por um erro num cálculo intermediário (o fator de conversão ano trópico/ano juliano foi omitido por Standish).

Com as unidades definidas acima, as matrizes $M_{11}, M_{12}$, $M_{21}$ e $M_{22}$, componentes da matriz $M$ de Murray, são a forma:

$$
\begin{aligned}
M_{11} & =X(0)+T_{J} \dot{X}(0), \\
M 12 & =T_{J} F X(0) / R \\
M_{21} & =R \dot{X}(0) \\
M_{22} & =F X(0),
\end{aligned}
$$

onde $T_{J}(=0,500002095577002)$ é o tempo decorrido entre $B 1950,0$ e J2000, 0 em séculos julianos, $F$ (= $1,000021359027778)$ o fator de conversão permitindo passar de um tempo em anos julianos para um tempo em anos trópicos e $R(=206264,8062470964)$ o fator de conversão permitindo passar de ângulos em radianos para ângulos em segundos de arco. É preciso notar que $X(0)$ representa o produto da matriz de rotação $R_{3}(-E(19,50))$, onde $E(T)$ vem dado pela equação (6), pela matriz de rotação $\mathrm{P}$ fazendo passar do equador e equinócio médios B1950, 0 ao equador e equinócios médios J2000, 0segundo Lieske 1979 [26], isto é:

$$
X(0)=P R 3(0 ", 525),
$$

O vetor "posição" de uma estrela a uma data qualquer $T$, levada ao plano fundamental e ao equinócio do $F K 5$, vale:

$$
\vec{r}+\frac{t}{R} \vec{v}=X(0)\left(\vec{r}_{1}+\frac{t}{R} \vec{v}\right)+F^{-1} t_{1} X(0) \vec{r}_{1}
$$

onde $t$ é o tempo em séculos julianos de $J 2000,0$ à data $T$ e $t_{1}$ o tempo em séculos trópicos de $B 1950,0$ à data $T$, com

$$
t=F^{-1} t_{1}-T_{J}
$$

A diferença entre as matrizes $M$ de Standish e de Murray, no sentido "Standish - Murray", vale: 


$$
\Delta M_{S, M}=\left(\begin{array}{ccc}
10^{-10}\left(\begin{array}{ccc}
0 & 21 & 46 \\
-21 & 0 & 1 \\
-46 & 0 & 0
\end{array}\right) & 10^{-13}\left(\begin{array}{ccc}
-518 & 6 & 3 \\
-6 & -518 & 0 \\
-3 & 0 & -518
\end{array}\right) \\
10^{-5}\left(\begin{array}{ccc}
2 & 88 & 188 \\
-88 & 1 & 2 \\
-188 & 0 & 1
\end{array}\right) & 10^{-8}\left(\begin{array}{ccc}
-2136 & 24 & 10 \\
-24 & -2136 & 0 \\
-10 & 0 & -2136
\end{array}\right)
\end{array}\right)
$$

As diferenças correspondentes às coordenadas e movimentos próprios são, respectivamente, de $0^{\prime \prime}, 01$ e $0^{\prime \prime}, 02$ por século para estrelas de grande movimento próprio $\left(1050^{\prime \prime}\right.$ por século). Estas diferenças diminuem com o movimento próprio.

A diferença $\triangle M_{A, M}$ entre as matrizes $M$ de Aoki et al. [19] e de Murray [24], no sentido "Aoki - Murray", vale:

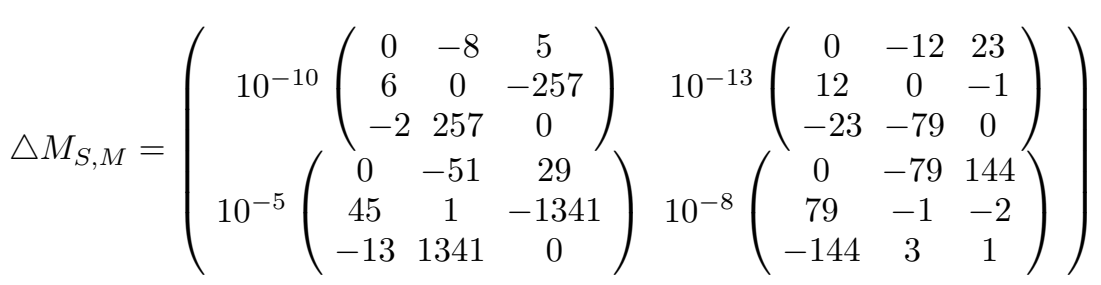

As diferenças correspondentes sobre as coordenadas e os movimentos próprios são, respectivamente, $0^{\prime \prime}, 005$ e 0", 013 por século, qualquer que seja o movimento próprio.

\section{RADIOFONTES}

Neste caso, a idéia é de calcular as ascensões retas e as declinações levadas ao plano fundamental e ao equinócio do $F K 5$ de radiofontes em função de suas coordenadas em $B 1950,0$ levadas ao plano e ao equinócio do $F K 4$. Iremos supor que os movimentos próprios no sistema $F K 5$ são nulos. Ainda dispomos de dois métodos.

\section{A. Método Merit (Melbourne 1983)}

a) determinamos a época média de observações consideradas, por exemplo, a época média de um catálogo de radiofontes;

b) depois, calculamos as coordenadas das radiofontes levadas ao equador e ao equinócio médios desta data com ajuda das fórmulas de precessão utilizadas para a construção do catálogo (em geral aquela de Newcomb) entre $B 1950,0$ e a data;

c) efetuamos a correção

$$
\delta \alpha=0^{s}, 0775+0^{s}, 0851 t+0^{s}, 0002 t^{2},
$$

onde $t$ é a época média das observações contadas em séculos julianos a partir de J2000, 0 . d) a partir das ascensões retas e das declinações obtidas, calculamos as coordenadas levadas ao equador e ao equinócio de referência do $F K 5$ com a ajuda da precessão de Lieske et al. [12] entre a data média das observações e J2000, 0;

e) finalmente, as coordenadas obtidas são, se necessárias, corrigidas da aberração elíptica.

\section{B. Método de Murray}

A direção da rádio fonte no sistema $F K 4$ é definida pelo vetor $\vec{r}_{0}$ eq. (8). A direção corrigida da aberração elíptica é definida pelo vetor $r_{1}(4,8$ ou 10$)$. O vetor "posição" da radiofonte levada ao plano fundamental e ao equinócio do FK5 é dada por:

$$
\mathbf{r}=X(0) \mathbf{r}_{1}
$$

onde $X(0)$ é dada pela expressão (18).

\section{POSIÇÕES DE CORPOS DO SISTEMA SOLAR}

Vamos discutir, rapidamente, duas situações:

\section{a) Fornecidas pelas observações}

Trata-se de transformar as observações de corpos do sistema solar reduzidas no sistema $F K 4$, com ajuda de estrelas do campo contidas no $F K 4$, em observações reduzidas no sistema $F K 5$. Um método aproximado foi proposto por Yeomans (1990) [27] para os cometas e os asteróides, mas ele se aplica igualmente aos planetas e satélites. 


\section{b) Fornecidas pelas efemérides}

Trata-se de passar das coordenadas de um planeta no instante $T$ levadas ao equador e ao equinócio de referência do $F K 4$ as coordenadas do planeta no mesmo instante levadas ao equador e ao equinócio de referência do FK5. Yeomans (1990) [27], propôs um método equivalente àquele de Murray para as radiofontes:

Vamos definir o vetor posição geocêntrico no sistema $F K 4$, levado ao plano fundamental e ao equinócio do $F K 4$ :

$$
\vec{r}_{0}=r_{0}\left(\begin{array}{c}
\cos \alpha_{0} \cos \delta_{0} \\
\sin \alpha_{0} \cos \delta_{0} \\
\sin \delta_{0}
\end{array}\right),
$$

onde $\vec{r}_{0}$ é a distância Terra-planeta. Trata-se de uma posição astrométrica no sentido anterior a 1984, incluindo, portanto, a aberração elíptica, que pode ser eliminada, por exemplo, através da expressão abaixo obtida a partir da expressão (17):

$$
\vec{r}_{1}=\vec{r}_{0}-\frac{1}{r_{0}}\left(\vec{r}_{0} \times\left(\vec{A} \times \vec{r}_{0}\right)\right)
$$

que é equivalente à expressão seguinte, deduzida de (15):

$$
\vec{r}_{1}=\vec{r}_{0}-r_{0} \vec{A}+\frac{\vec{r}_{0}}{r_{0}}\left(\vec{A} \cdot \vec{r}_{0}\right)
$$

onde $\vec{A}$ é dada por (14).

$\mathrm{O}$ vetor posição geocêntrico no sistema $F K 5$, levado ao plano fundamental e ao equinócio do $F K 5$ :

$$
\vec{r}=r\left(\begin{array}{c}
\cos \alpha_{0} \cos \delta_{0} \\
\sin \alpha_{0} \cos \delta_{0} \\
\sin \delta_{0}
\end{array}\right),
$$

é dado pela expressão

$$
\vec{r}=X(0) \vec{r}_{1}
$$

O vetor velocidade levado ao plano fundamental e ao equinócio do $F K 5$ será dado por:

$$
\vec{v}_{0}=X(0) \vec{v}_{1}
$$

sendo $\vec{v}_{0}$ o vetor velocidade levado ao plano fundamental e ao equinócio do $F K 4$.

\section{CONCLUSÕES}

O nosso objetivo neste trabalho é apresentar, de maneira acessível a um aluno de graduação em Física, uma discussão sobre algumas bases de referência utilizadas em Astronomia. Adotamos um cenário histórico e um tratamento matemático. Se, por um lado, vários cursos de bacharelado em Física já admitem uma concentração em temas de Astronomia, por outro lado, a despeito dos ótimos textos já existentes em português, em nenhum deles há a preocupação em apresentar uma visão rigorosa do assunto.

Este texto foi escrito tendo em mente um aluno que já tenha feito um curso introdutório de Astronomia Fundamental, podendo o mesmo ser usado como notas de aula em um curso de Astrometria, por exemplo.
[1] P.C.R. Poppe, V.A.F. Martin; Sobre os Sistemas de Referência Celeste, Boletim da SAB 22, (2) (2002).

[2] E.M. Standish, J.G. Williams; Numerical Integration DE200/LE200, (1982).

[3] H. Eichhorn; Astronomy of Star Positions, Frederick Ungar, New York, (1974).

[4] H.G. Walter, O.J. Sovers; Astrometry of Fundamental Catalogues. The Evolution from optical to radio reference frames, Springer-Verlag, (2000).

[5] F. Küstner; Beobachtete Correctionen des FundamentalCataloges von Auwers, Astron. Nachrichten 158129 (1902).

[6] A. Kahrstedt; Über die Verbesserung des Äquinoktiums des NFK, Astron. Nachrichten 24433 (1931).

[7] W. Fricke; Fundamental Catalogues, Past, Present and Future, Celestial Mechanics, 36207 (1985).

[8] A. Kopff et al.; FK4. Veröffentlichungen Astgronomisches, Rechen-Institut, 14 (1963).

[9] W. Gliese; Veröffentlichungen Astgronomisches, RechenInstitut, 12 (1963).

[10] W. Fricke, H. Schwan, T. Lederle; FK5, Veröffentlichungen Astgronomisches, Rechen-Institut, 32 (1988).

[11] W. Fricke; Determination of the Equinox and Equator of the FK5, A \& A 107, L13 (1982).

[12] J.H. Lieske et al.; Expression for the Precession quantities based upon the IAU (1976) System of Astronomical Constants, A \& A 581 (1977).

[13] S. Röser, U. Bastian; PPM Star Catalogue, Spectrum Akademisher Verlag, (1991).
[14] L. Lindegren; Status on early results of the Hipparcos astrometry project. European International Space Year Conference, Munich (april). Satellite Symposium 3, Space Sciences with particular emphasis on High Energy Astrophysics, (1992).

[15] P. Lacroûte; Trans. IAU, XIII B 63 (1968).

[16] ESA; The Hipparcos and TYCHO Catalogues. ESA SP1200, European Space Agency, (1997).

[17] E.M. Standish; Orientation of the JPL Ephemerides DE200/LE200 to the Dynamical Equinox of J2000, A \& A 114297 (1982).

[18] J. Kovalevsky, I.I. Mueller; Introduction in Reference Frames in Astronomy and Geophysics, J. Kovalevsky, I.I. Muller and B. Kolaczek (eds.), Kluwer, Dordrecht, 1 (1989).

[19] S. Aoki, M. Sôma, H. Kinoshita, K. Inoue; Conversion matrix of epoch B1950, 0 FK4-based positions of stars to epoch J2000, 0 in accordance with the new IAU resolutions, A \& A 128263 (1983).

[20] W. Fricke; Arguments in favour of a change in precession, A \& A 54363 (1977).

[21] E.F. Arias, M. Feissel, J.F. Lestrade; The IERS extragalactic celestial reference frame and its tie with Hipparcos, IERS Technical Note (7), Observatoire de Paris, (1991).

$[22]$ W. Fricke; Determinations of positions and the reference system, Mittelungen B109 43 (1982).

[23] E.M. Standish; Conversion of positions and proper motions from B1950, 0 to the IAU system at J2000,0, A \& A 115 20 (1982). 
[24] C.A. Murray; The transformation of coordinates between the systems of integrated ephemeris of the Moon and planets spanning forty-four centuries, A \& A 125150 (1989).

[25] W. Melbourne (ed.); Project Merit Standards, USNO Circular (167), USNO Washington D.C., (1983).
[26] J.H. Lieske; Precession matrix based on IAU (1976) system of astronomical constants, A \& A 73282 (1979).

[27] D.K. Yeomans; BAAS 22946 (1990). 